\title{
Calculation of partial safety factors of breakwater armor stones considering correlation between wave height and wave steepness
}

\author{
Seung-Woo Kim and Kyung-Duck Suh* \\ Department of Civil and Environmental Engineering, Seoul National University, 599 \\ Gwanangno, Gwanak-gu, Seoul 151-744, Republic of Korea \\ *kdsuh@snu.ac.kr
}

\begin{abstract}
In the calculation of partial safety factors of breakwater armor stones, it has been assumed that all the design variables are independent one another. However, some of them are not independent but are correlated each other. In the present study, the partial safety factors are calculated by considering the correlation between wave height and wave steepness. Smaller partial safety factors and smaller armor weight are obtained if the correlation is taken into account. The reduction becomes prominent as the probability of failure decreases (or the design armor weight increases). The correlation between wave height and steepness in real sea is also estimated by using the wave hindcasting data along the Korean coast.
\end{abstract}

Keywords: armor stones, breakwaters, correlation, partial safety factors, reliability-based design, wave height, wave steepness

\section{Introduction}

During the last several decades, the design method of civil engineering structures has been changed from the conventional allowable stress design to the limit state design, which is also called partial safety factor design or load and resistance factor design. In the allowable stress design, the safety of a structure is obtained by using the safety factor for considering the uncertainties of load and resistance. These uncertainties should be considered in the limit state design as well. The safety factor in the allowable stress design

\footnotetext{
* Corresponding author.
} 
is determined more or less arbitrarily based on the experience and judgment of engineers. In the limit state design, however, the uncertainties of load and resistance can be considered more reasonably and objectively by performing reliability analyses based on the statistical characteristics of design variables.

In the design of breakwaters, van der Meer (1988) proposed a probabilistic approach for the design of breakwater armor layers, and Burcharth (1991) introduced the partial safety factors in the design of rubble mound breakwaters. Later Burcharth and Sørensen (2000) established partial safety factor systems for rubble mound breakwaters and vertical breakwaters by summarizing the results of the PIANC (Permanent International Association of Navigational Congresses) Working Group. They calculated the partial safety factors on the assumption that all design variables are independent one another. However, some of them are not independent but are correlated each other. For instance, in the van der Meer (1987) formula for breakwater armor stones attacked by plunging waves, wave height and wave steepness are correlated each other. In the present study, we calculate the partial safety factors of breakwater armor stones considering the correlation between wave height and steepness, and compare them with those of Burcharth (1992) who did not consider the correlation. We also estimate the correlation between wave height and steepness in real sea using the wave hindcasting data along the Korean coast.

\section{Correlation between design variables}

\subsection{Correlation coefficient of random variables}

In order to calculate the influence factor using correlated random variables, we have to calculate the correlation coefficient. The covariance matrix of correlated random variables, $X_{1}, X_{2}, \cdots, X_{n}$, is calculated as

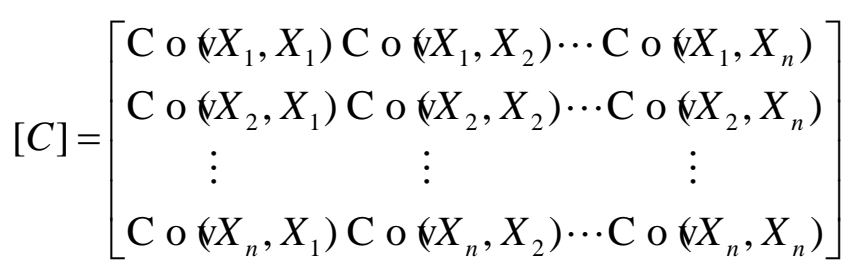

where $\operatorname{Cov}\left(X_{i}, X_{j}\right)$ is the covariance between random variables $X_{i}$ and $X_{j}$. The 
covariance between normalized random variables $X_{i}^{\prime}$ and $X_{j}^{\prime}$ is expressed as

$$
\begin{aligned}
\operatorname{Cov}\left(X_{i}^{\prime}, X_{j}^{\prime}\right) & =E\left[\left(X_{i}^{\prime}-\mu_{X_{i}}{ }^{\prime}\right)\left(X_{j}^{\prime}-\mu_{X_{j}}{ }^{\prime}\right)\right] \\
& =\frac{E\left[\left(X_{i}-\mu_{X_{i}}\right)\left(X_{j}-\mu_{X_{j}}\right)\right]}{\sigma_{X_{i}} \sigma_{X_{j}}}=\frac{\operatorname{Co}\left(X_{i}, X_{j}\right)}{\sigma_{X_{i}} \sigma_{X_{j}}}=\rho_{X_{i} X_{j}}
\end{aligned}
$$

where $E[\bullet]$ denotes the expectation, $\mu_{X_{i}}$ and $\sigma_{X_{i}}$ are respectively the mean and standard deviation of the random variable $X_{i}$, and $\rho_{X_{i} X_{j}}$ is the correlation coefficient between $X_{i}$ and $X_{j}$. The normalization method of a random variable will be explained in Section 3.3. The preceding equation describes that the covariance between normalized random variables $X_{i}{ }^{\prime}$ and $X_{j}{ }^{\prime}$ is the same as the correlation coefficient between $X_{i}$ and $X_{j}$. Therefore, the covariance matrix of normalized random variables, $X_{1}{ }^{\prime}, X_{2}{ }^{\prime}, \cdots, X_{n}{ }^{\prime}$, is calculated as

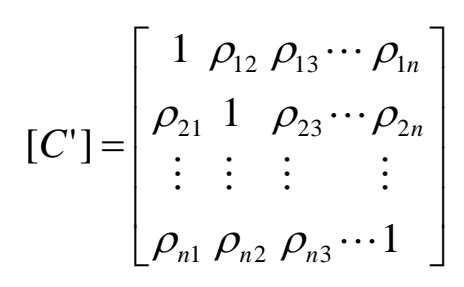

Using the orthogonal transformation matrix $\mathbf{T}$ composed of the eigenvectors of the matrix $\left[C^{\prime}\right]$, the correlated random variables can be transformed into non-correlated variables by

$$
\mathbf{Y}=\mathbf{T}^{t} \mathbf{X}^{\prime}
$$

where $\mathbf{X}^{\prime}=\left(X_{1}{ }^{\prime}, X_{2}{ }^{\prime}, \cdots, X_{n}{ }^{\prime}\right)$ is the normalized correlated random variables, $\mathbf{Y}=\left(Y_{1}, Y_{2}, \cdots, Y_{n}\right)$ is the transformed non-correlated variables, and the superscript $t$ 
denotes a transpose matrix. Since $\mathbf{T}$ is an orthogonal matrix, its inverse $\mathbf{T}^{-1}$ is the same as $\mathbf{T}^{t}$, and hence $\mathbf{X}, \mathbf{X}^{\prime}$, and $\mathbf{Y}$ are related as follows:

$$
\mathbf{X}=\left[\sigma_{\mathbf{X}}\right] \mathbf{X}^{\prime}+\mu_{\mathbf{X}}=\left[\sigma_{\mathbf{X}}\right] \mathbf{T Y}+\mu_{\mathbf{X}}
$$

where $\mathbf{X}^{\prime}=\mathbf{T Y}$, and $\left[\sigma_{\mathbf{X}}\right]$ and $\mu_{\mathbf{X}}$ are given by

$$
\begin{gathered}
{\left[\sigma_{\mathbf{X}}\right]=\left[\begin{array}{cccc}
\sigma_{X_{1}} & 0 & \cdots & 0 \\
0 & \sigma_{X_{2}} & \cdots & 0 \\
\vdots & \vdots & & \vdots \\
0 & 0 & \cdots & \sigma_{X_{n}}
\end{array}\right]} \\
\mu_{\mathbf{x}}=\left(\mu_{X_{1}}, \mu_{X_{2}}, \cdots, \mu_{X_{n}}\right)
\end{gathered}
$$

respectively. On the other hand, the covariance of the random variable $\mathbf{Y}$ is given by

$$
\left[C_{\mathbf{Y}}\right]=E\left(\mathbf{Y} \mathbf{Y}^{t}\right)=E\left(\mathbf{T}^{t} \mathbf{X}^{\prime} \mathbf{X}^{t} \mathbf{T}\right)=\mathbf{T}^{t} E\left(\mathbf{X}^{\prime} \mathbf{X}^{\prime t}\right) \mathbf{T}=\mathbf{T}^{t}\left[C^{\prime}\right] \mathbf{T}=[\lambda]
$$

The preceding equation states that the eigenvalue $[\lambda]$ of the matrix $\left[C^{\prime}\right]$ is the same as the covariance of the random variable $\mathbf{Y}$.

\subsection{Calculation of correlation coefficient}

The design formula proposed by van der Meer (1987) for plunging waves can be written in the form including partial safety factors as

$$
D_{n 50} \geq \frac{\gamma_{S} \gamma_{R} H_{s}}{A_{v} S_{d}^{0.2} P^{0.18} \Delta(\cot \alpha)^{0.5} s_{0 m}^{0.25} N_{w}^{-0.1}}
$$

where $D_{n 50}$ is the equivalent cube length of median armor stone, which is the side length of a cube having the same volume as the armor stone, $H_{s}$ is the design significant wave height, $A_{v}$ is the coefficient representing the uncertainty of the empirical formula, $S_{d}$ is the damage level, which is defined as the eroded area divided by $D_{n 50}^{2}, P$ is the 
permeability coefficient underneath the armor layer, $\Delta=\rho_{s} / \rho_{w}-1, \rho_{s}$ and $\rho_{w}$ are mass density of rock and water, respectively, $\alpha$ is the slope angle of breakwater, $s_{0 m}=H_{s} / L_{0 m}$ is the wave steepness, $L_{0 m}$ is the deepwater wavelength corresponding to mean wave period, $N_{w}$ is the number of waves during a storm, and $\gamma_{S}$ and $\gamma_{R}$ are the partial safety factors of load and resistance, respectively. In Eq. (9), all the variables are assumed to be independent one another except between $H_{s}$ and $s_{0 m}$.

In order to calculate the correlation coefficient between $H_{s}$ and $s_{0 m}$, we used the data of Lee and Jun (2006), who established a data base of hindcasted wave parameters such as significant wave height, peak period and direction for the period of 25 years starting from 1979 and for major 106 typhoons for 53 years since 1951 at each grid point of the northeast Asia regional seas with grid size of $18 \mathrm{~km}$. The HYPA (HYbrid PArametrical) model and the ECMWF (European Center for Medium-range Weather Forecasts) wind data were used for the simulation of waves for the extra-tropical storms, while the WAM model was used for the simulation of typhoon waves using the wind field calculated by a typhoon wind model with carefully analyzed typhoon parameters. They also presented the design wave heights for return period of 50 years at the 106 coastal grid points around the Korean peninsula as indicated as black dots in Fig. 1. In this study, we used the annual maximum wave heights and the corresponding wave periods during 25 years from 1979 to 2003 (including typhoon wave data for the same period) at the coastal grid points. The coastal grid points are divided into three regions as shown in Fig. 1 depending on wave characteristics. The large waves along the east and west coasts of Korea (Region I and III) are usually generated by extra-tropical storms in winter and spring, while the southern part of Korea (Region II) is influenced by large typhoon waves in summer and fall.

Fig. 2 shows the relationship between significant wave height and steepness on each coast. The curve-fitted line and correlation coefficient are also given. The correlation coefficient is almost same on the east and south coasts even though the curve-fitted lines are different, while it is small on the west coast. On the other hand, the wave steepness has the mean of $0.030,0.035$, and 0.033 , and standard deviation of $0.011,0.013$, and 0.008 on the east, south, and west coasts, respectively. It is observed some data in Fig. 2 are lined on the radial lines. This is because the periods of typhoon waves refer to the spectral peak period rather than the mean wave period. The spectral peak period, $T_{p}$, was converted to the mean period by the relationship, $T_{m}=T_{p} / 1.21$. In the WAM model, a sparse 
frequency resolution is used to calculate wave interactions among different frequency components. If the mean period was calculated by $T_{m}=\sqrt{m_{0} / m_{2}}$, where $m_{0}$ and $m_{2}$ respectively are the zero-th and second moments of the frequency spectrum, the lining problem could be avoided.

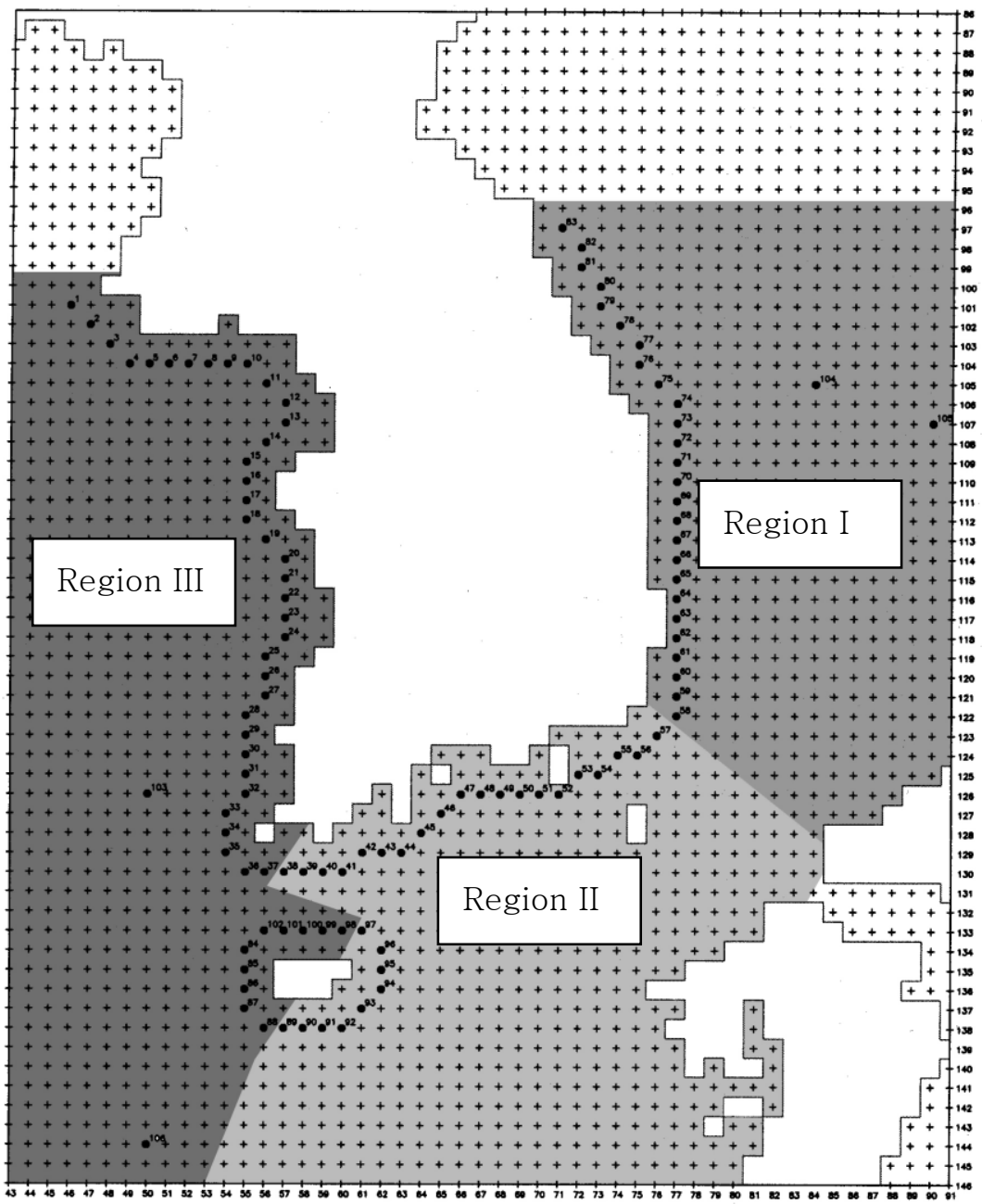

Fig. 1. Coastal grid points for design wave height estimation and regions divided depending on wave characteristics 


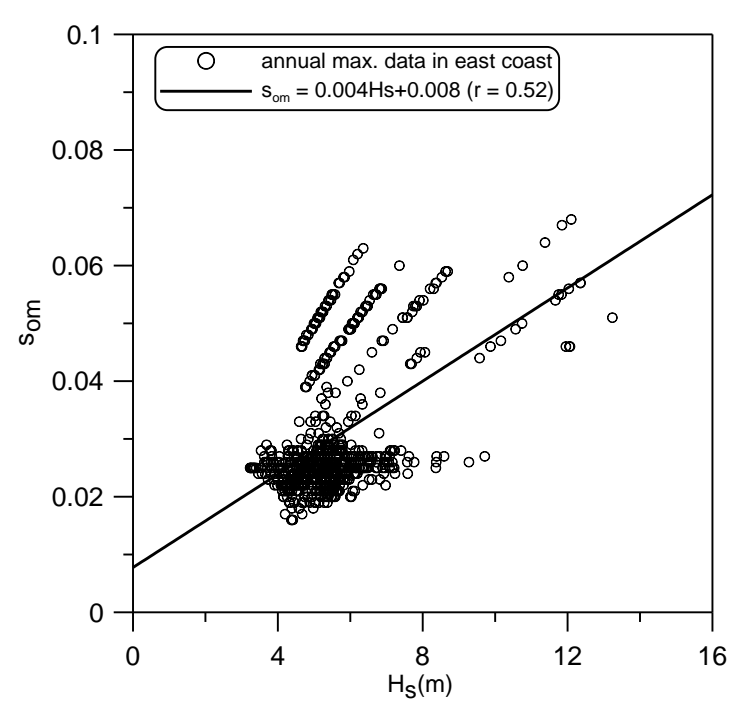

(a) East coast (Region I)

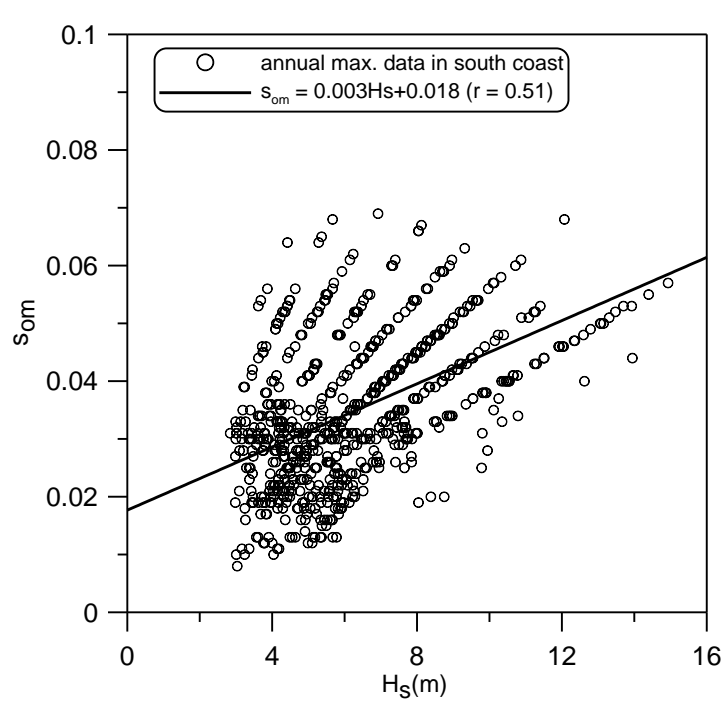

(b) South coast (Region II)

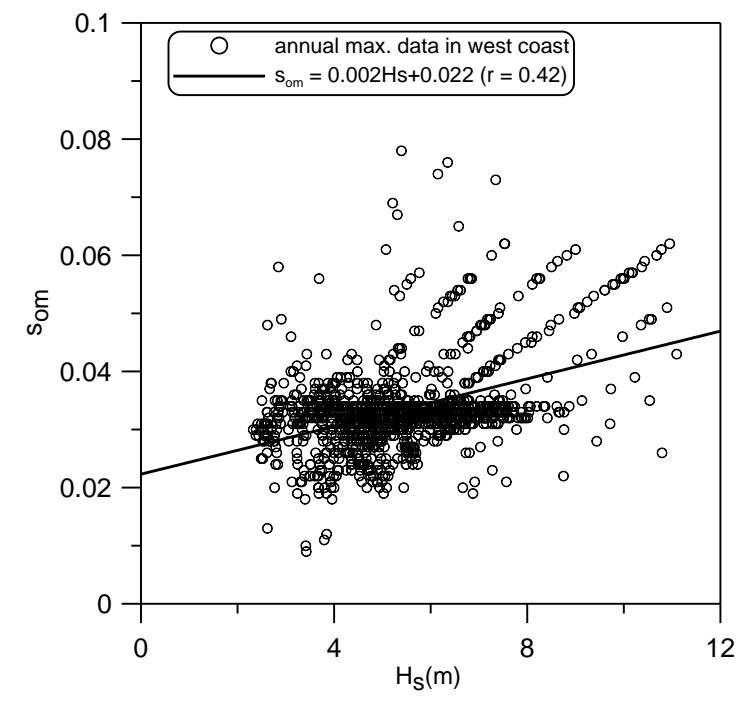

(c) West coast (Region III)

Fig. 2. Relationship between significant wave height and wave steepness along the coasts of Korea 


\section{Reliability analysis}

In order to compare the calculated partial safety factors with those of Burcharth (1992), we used the same statistical characteristics of design variables as his as given in Table 1. In the table, $\delta$ denotes the coefficient of variation, and $k, A$, and $B$ are shape, scale, and location parameters, respectively. $v$ is the number of storm events in a year.

In the case of the south coast of Korea, considering the correlation between wave height and steepness, the covariance matrix of the normalized variables $\mathbf{X}^{\prime}=\left(A_{v}{ }^{\prime}, D_{n}{ }^{\prime}, \cdots, H_{s}{ }^{\prime}\right)$ is expressed as

$$
\left[C^{\prime}\right]=\left[\begin{array}{cccccc}
1 & 0 & 0 & \cdots & 0 & 0 \\
0 & 1 & 0 & \cdots & 0 & 0 \\
\vdots & \vdots & \vdots & \cdots & \vdots & \vdots \\
0 & 0 & 0 & \cdots & 1 & 0.51 \\
0 & 0 & 0 & \cdots & 0.51 & 1
\end{array}\right]
$$

Table 1. Statistical characteristics of design variables of van der Meer (1987) formula.

\begin{tabular}{cccccc}
\hline No. & $X_{i}$ & $\mu_{X_{i}}$ & $\sigma_{X_{i}}$ & $\delta_{X_{i}}$ & Distribution \\
\hline 1 & $A_{v}$ & 6.2 & 0.4 & 0.065 & Normal \\
2 & $D_{n 50}$ & various & various & 0.030 & Normal \\
3 & $\Delta$ & 1.72 & 0.054 & 0.031 & Normal \\
4 & cot $\alpha$ & 1.50 & 0.075 & 0.050 & Normal \\
5 & $P$ & 0.40 & 0.040 & 0.100 & Normal \\
6 & $N_{w}$ & 2500 & 1250 & 0.500 & Normal \\
7 & $s_{0 m}$ & 0.04 & 0.010 & 0.250 & Normal \\
\cline { 3 - 4 } 8 & $H_{s}$ & $k=1.39, v=4.17, A=1.06, B=0.44$ & Weibull \\
\hline
\end{tabular}


The diagonal matrix $[\lambda]$ consisted of the eigenvalues of the matrix $\left[C^{\prime}\right]$ is given as the covariance of the non-correlated variable $\mathbf{Y}$, i.e.,

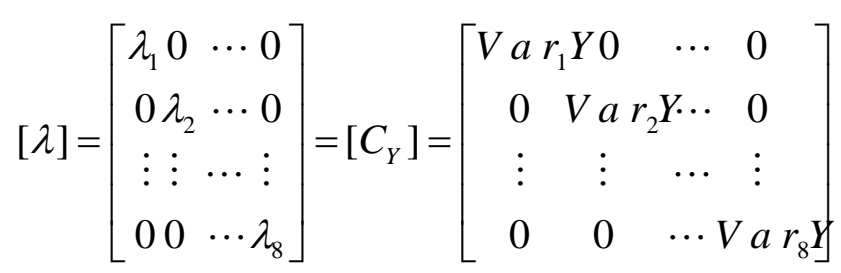

where $\lambda_{i}=1.0$ for $i=1$ to $6, \lambda_{7}=0.49, \lambda_{8}=1.51$, and $\operatorname{Var}$ denotes the variance. The orthogonal transformation matrix obtained by calculating the eigenvectors corresponding to each eigenvalue is given by

$$
\mathbf{T}=\left[\begin{array}{cccccccc}
1 & 0 & 0 & 0 & 0 & 0 & 0 & 0 \\
0 & 1 & 0 & 0 & 0 & 0 & 0 & 0 \\
\vdots & \vdots & \vdots & \vdots & \vdots & \vdots & \vdots & 0 \\
0 & 0 & 0 & 0 & 0 & 1 & 0 & 0 \\
0 & 0 & 0 & 0 & 0 & 0 & 1 / \sqrt{2} & 1 / \sqrt{2} \\
0 & 0 & 0 & 0 & 0 & 0 & -1 / \sqrt{2} & 1 / \sqrt{2}
\end{array}\right]
$$

In this study, we use the Level 2 FORM (First-Order Reliability Method) for the reliability analysis of armor stones. Usually an iterative method is used to achieve the convergence of influence factors and design point coordinates.

\subsection{Calculation of influence factors}

The reliability function of the stability formula for plunging waves of van der Meer (1987) is given by

$$
Z=A_{v} S_{d}^{0.2} D_{n 50} \Delta \sqrt{\cot \alpha} P^{0.18} N_{w}^{-0.1} S_{0 m}^{0.25}-H_{s}^{T}
$$

where $H_{s}^{T}$ is the design significant wave height for $T$-year lifetime of the breakwater. 
Since $s_{0 m}$ and $H_{s}^{T}$ are correlated, the preceding reliability function cannot be used in its form, and it should be expressed in terms of non-correlated variables. For this, the correlated design variables in Table 1 are transformed into the non-correlated variables $\mathbf{Y}$ using Eqs. (5) and (12):

$$
\begin{aligned}
& A_{v}=\sigma_{A_{v}} Y_{1}+\mu_{A_{v}} \\
& D_{n 50}=\sigma_{D_{n}} Y_{2}+\mu_{D_{n 50}} \\
& \Delta=\sigma_{\Delta} Y_{3}+\mu_{\Delta} \\
& \operatorname{c~o~d~}=\sigma_{\text {c oct }} Y_{4}+\mu_{\mathrm{coct}} \\
& P=\sigma_{P} Y_{5}+\mu_{P} \\
& N_{w}=\sigma_{N_{w}} Y_{6}+\mu_{N_{w}} \\
& s_{0 m}=\sigma_{s_{0 m}}\left(Y_{7}+Y_{8}\right) / \sqrt{2}+\mu_{s_{0 m}} \\
& H_{s}^{T}=\sigma_{H_{s}^{T}}\left(-Y_{7}+Y_{8}\right) / \sqrt{2}+\mu_{H_{s}^{T}}
\end{aligned}
$$

Substituting these equations into Eq. (13), the reliability function is expressed in terms of non-correlated variables $\mathbf{Y}$ :

$$
\begin{aligned}
Z= & \left(\sigma_{A_{v}} Y_{1}+\mu_{A_{v}}\right) S_{d}^{0.2}\left(\sigma_{D_{n 50}} Y_{2}+\mu_{D_{n 50}}\right)\left(\sigma_{\Delta} Y_{3}+\mu_{\Delta}\right) \sqrt{\sigma_{\cot \alpha} Y_{4}+\mu_{\cot \alpha}}\left(\sigma_{P} Y_{5}+\mu_{P}\right)^{0.18} \\
& \times\left(\sigma_{N_{w}} Y_{6}+\mu_{N_{w}}\right)^{-0.1}\left[\sigma_{s_{0 m}}\left(Y_{7}+Y_{8}\right) / \sqrt{2}+\mu_{s_{0 m}}\right]^{0.25}-\left[\sigma_{H_{s}^{T}}\left(-Y_{7}+Y_{8}\right) / \sqrt{2}+\mu_{H_{s}^{T}}\right]
\end{aligned}
$$

Applying the Rackwitz (1976) algorithm to the preceding equation, the influence factors are calculated as

$$
\alpha_{Y_{i}}=\frac{-\left(\frac{\partial Z}{\partial Y_{i}^{\prime}}\right)_{*}}{\sqrt{\sum_{i=1}^{8}\left(\frac{\partial Z}{\partial Y_{i}^{\prime}}\right)_{*}^{2}}}
$$


where the prime indicates a normalized value, and * denotes the design point, i.e., the most probable failure point.

\subsection{Comparison of calculated influence factors}

In this section, we compare the influence factors calculated with and without considering the correlation between wave height and steepness. The correlation coefficient on the south coast of Korea was used, i.e., $\rho_{H_{s}, s_{0 m}}=0.51$.

The rightmost column of Table 2 shows the influence factors calculated without considering the correlation. Only two times of iteration were needed for the convergence of the reliability index, which will be explained in the following sub-section. The positive and negative values represent resistance and load variables, respectively. The variable of the greatest influence in the design is the wave height, and it is followed by the empirical coefficient of the formula and the wave steepness of almost same degree of importance. Table 3 shows the influence factors calculated considering the correlation. Compared with the results in Table 2, the magnitudes of the influence factors of all the design variables increased except the wave height, the magnitude of the influence factor of which decreased.

\subsection{Calculation of reliability index}

The design variables are normalized using the influence factors as follows:

$$
X_{i}{ }^{\prime}=-\beta \alpha_{X_{i}}
$$

The variable at the design point $P$ is then expressed as

$$
\left.X_{i}\right|_{P}=\sigma_{X_{i}} X_{i}{ }^{\prime}+\mu_{X_{i}}=\mu_{X_{i}}-\alpha_{X_{i}} \sigma_{X_{i}} \beta
$$

This equation is substituted for each variable in Eq. (13) and is calculated the reliability index $\beta$ that makes $Z=0$. Iteration was made until the difference from the previous $\beta$ 
is less than 0.005 . The final reliability index is substituted into the following equation to calculate the probability of failure as

Table 2. Iterative calculation of reliability index without considering correlation.

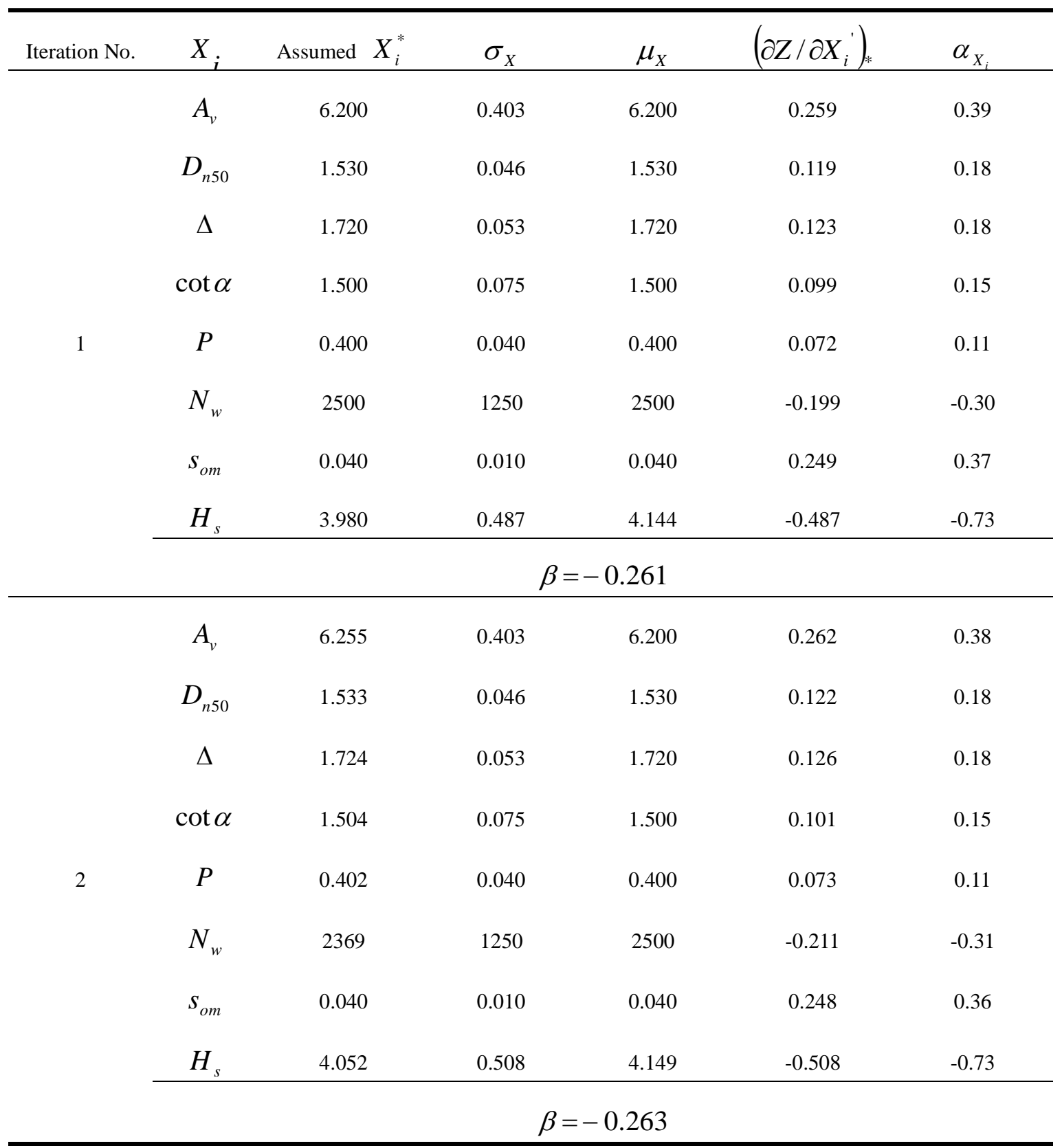


Table 3. Iterative calculation of reliability index considering correlation.

\begin{tabular}{|c|c|c|c|c|c|c|c|c|}
\hline Iteration No. & $X_{i}$ & Assumed $X_{i}^{*}$ & $\sigma_{X}$ & $\mu_{X}$ & $Y_{i}$ & $Y_{i}^{*}$ & $\left(\partial Z / \partial Y_{i}^{\prime}\right)_{*}$ & $\alpha_{Y_{i}}$ \\
\hline \multirow{9}{*}{1} & $A_{v}$ & 6.200 & 0.403 & 6.200 & $Y_{1}$ & 0.000 & 0.259 & 0.45 \\
\hline & $D_{n 50}$ & 1.530 & 0.046 & 1.530 & $Y_{2}$ & 0.000 & 0.119 & 0.21 \\
\hline & $\Delta$ & 1.720 & 0.053 & 1.720 & $Y_{3}$ & 0.000 & 0.123 & 0.22 \\
\hline & $\cot \alpha$ & 1.500 & 0.075 & 1.500 & $Y_{4}$ & 0.000 & 0.099 & 0.17 \\
\hline & $P$ & 0.400 & 0.040 & 0.400 & $Y_{5}$ & 0.000 & 0.072 & 0.13 \\
\hline & $N_{w}$ & 2500 & 1250 & 2500 & $Y_{6}$ & 0.000 & -0.199 & -0.35 \\
\hline & $s_{o m}$ & 0.040 & 0.010 & 0.040 & $Y_{7}$ & 0.241 & 0.520 & 0.64 \\
\hline & $H_{s}$ & 3.980 & 0.487 & 4.144 & $Y_{8}$ & -0.241 & -0.168 & -0.36 \\
\hline & \multicolumn{8}{|c|}{$\beta=-0.300$} \\
\hline \multirow{9}{*}{2} & $A_{v}$ & 6.255 & 0.403 & 6.200 & $Y_{1}$ & 0.136 & 0.262 & 0.44 \\
\hline & $D_{n 50}$ & 1.533 & 0.046 & 1.530 & $Y_{2}$ & 0.063 & 0.122 & 0.21 \\
\hline & $\Delta$ & 1.724 & 0.053 & 1.720 & $Y_{3}$ & 0.065 & 0.126 & 0.21 \\
\hline & $\cot \alpha$ & 1.504 & 0.075 & 1.500 & $Y_{4}$ & 0.052 & 0.101 & 0.17 \\
\hline & $P$ & 0.402 & 0.040 & 0.400 & $Y_{5}$ & 0.038 & 0.073 & 0.12 \\
\hline & $N_{w}$ & 2369 & 1250 & 2500 & $Y_{6}$ & -0.105 & -0.214 & -0.36 \\
\hline & $s_{o m}$ & 0.040 & 0.010 & 0.040 & $Y_{7}$ & 0.136 & 0.539 & 0.64 \\
\hline & $H_{s}$ & 4.052 & 0.508 & 4.149 & $Y_{8}$ & -0.136 & -0.180 & -0.37 \\
\hline & \multicolumn{8}{|c|}{$\beta=-0.303$} \\
\hline
\end{tabular}




$$
P_{f}=1-\Phi(\beta)
$$

where $\Phi$ is the cumulative distribution function of the normal distribution.

Fig. 3 shows the probability of failure for the weight of armor stones varying from 12 to 40 tons. The damage level $S_{d}=2.0$ was used as the criterion of failure, which is corresponding to the onset of damage (van der Meer, 1987). The lifetime of the breakwater $T=50$ years was used. In other words, $H_{s}^{50}$ was used in Eq. (13). Considering the correlation makes the probability of failure decrease. Therefore, a smaller weight of armor stones can be used if the correlation is considered in the design. The difference of the weight of armor stones between correlation and non-correlation increases as the probability of failure decreases. Therefore, if the correlation is considered, more reduction of armor weights can be made as the design weight increases (or the probability of failure decreases). For example, the weight reduces by only 2 tons (from 23 to 21 ton) for the probability of failure of $10 \%$, while it reduces by 4 tons (from 29 to 25 ton) for that of $5 \%$.

To explain the reason why the probability of failure decreases if the correlation is taken into account, let us consider a simple reliability function

$$
Z=R-S
$$

where $R$ and $S$ are the resistance and loading functions, respectively. If $R$ and $S$ are normally distributed and correlated, then the reliability function is also normally distributed with mean value

$$
\mu_{Z}=\mu_{R}-\mu_{S}
$$

and standard deviation

$$
\sigma_{Z}=\sqrt{\sigma_{R}^{2}+\sigma_{S}^{2}-2 \rho_{R S} \sigma_{R} \sigma_{S}}
$$

The reliability index is expressed as 


$$
\beta=\frac{\mu_{Z}}{\sigma_{Z}}=\frac{\mu_{R}-\mu_{S}}{\sqrt{\sigma_{R}^{2}+\sigma_{S}^{2}-2 \rho_{R S} \sigma_{R} \sigma_{S}}}
$$

Therefore, as the correlation coefficient increases, the reliability index increases, and the probability of failure decreases.

\section{Calculation of partial safety factors}

Eq. (13) can be simply expressed using the resistance function $R(\vec{X})$ and load function $S(\vec{Y})$ as

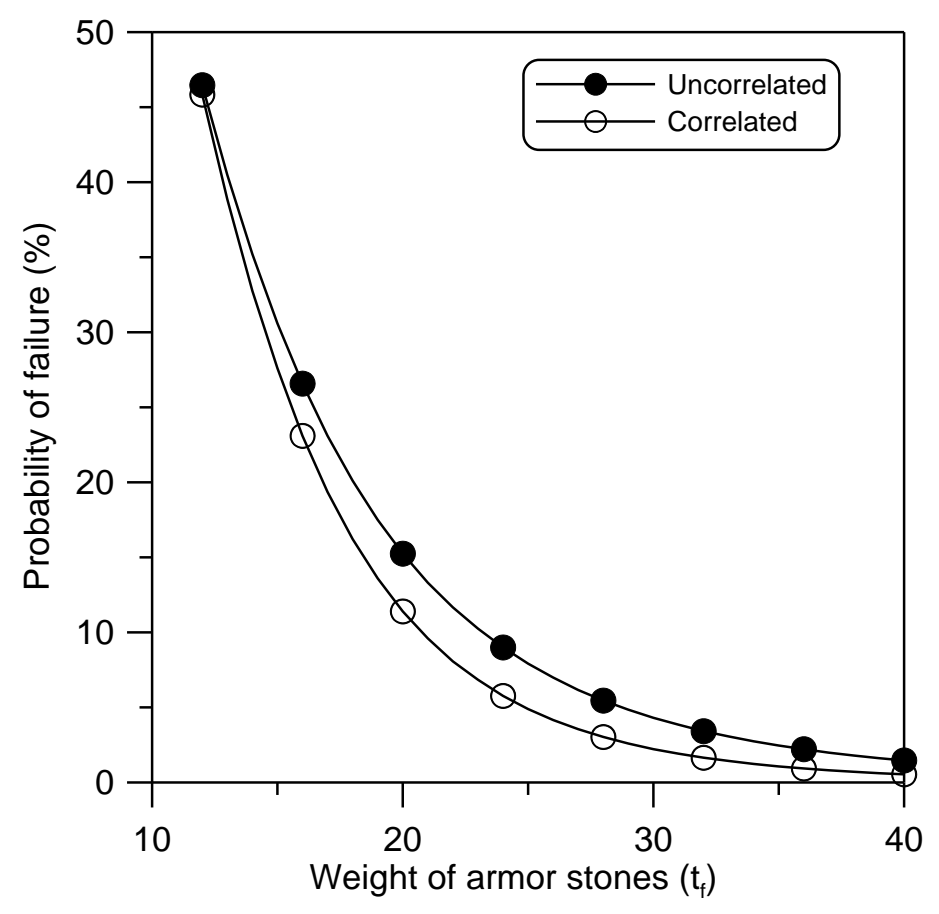

Fig. 3. Probability of failure versus weight of armor stone 


$$
Z(\vec{X}, \vec{Y})=R(\vec{X})-S(\vec{Y})
$$

where $\vec{X}$ and $\vec{Y}$ are the design variables included in the resistance and load functions, respectively. The design equation including the partial safety factors of load and resistance,

$\overrightarrow{\gamma_{S}}$ and $\overrightarrow{\gamma_{R}}$, is then expressed as

$$
\frac{R_{c}\left(\overrightarrow{X_{c}}\right)}{\overrightarrow{\gamma_{R}}} \geq \overrightarrow{\gamma_{S}} S_{c}\left(\overrightarrow{Y_{c}}\right)
$$

where the subscript $c$ denotes a characteristic value. In this study, the mean value is used as the characteristic value of each variable except the significant wave height for which the $T$-year return period value of $H_{s}$ is used. If the target reliability index $\beta_{T}$ is greater than 0.0 , the partial safety factors are greater than 1.0.

In order to calculate the partial safety factors, the design point (or the most probable failure point) must be calculated such that $Z(\vec{X}, \vec{Y})=0$ for the target reliability index $\beta_{T}$. To satisfy this, the design point and the characteristic values must have the following relationship:

$$
Z\left(\overrightarrow{X^{*}}\left(\beta_{T}\right), \overrightarrow{Y^{*}}\left(\beta_{T}\right)\right)=Z\left(\frac{\overrightarrow{X_{c}}}{\overrightarrow{\gamma_{R}}}, \overrightarrow{\gamma_{S}} \overrightarrow{Y_{c}}\right)
$$

In other words, the design variables at the design point are expressed as the product of the characteristic value and the partial safety factor. Therefore, the partial safety factors of resistance and load are expressed as

$$
\overrightarrow{\gamma_{R}}=\frac{\overrightarrow{X_{c}}}{\overrightarrow{X^{*}}\left(\beta_{T}\right)}, \quad \overrightarrow{\gamma_{S}}=\frac{\overrightarrow{Y^{*}}\left(\beta_{T}\right)}{\overrightarrow{Y_{c}}}
$$


In our problem, we have one load variable, $H_{s}$, and seven resistance variables, see Table 1. In fact, $N_{w}$ is also a load variable, but it is included in the resistance term for convenience' sake, with a negative exponent. It is common to use overall safety factors, like $\gamma_{S}$ and $\gamma_{R}$ in Eq. (9), which are obtained as the product of the partial safety factors calculated for each variable, i.e.,

$$
\begin{aligned}
& \gamma_{S}=\gamma_{H_{s}} \\
& \gamma_{R}=\gamma_{A_{v}} \gamma_{D_{n}} \gamma_{\Delta} \gamma_{\cot \alpha}^{0.5} \gamma_{P}^{0.18} \gamma_{S_{0 m}}^{0.25} \gamma_{N_{w}}^{-0.1}
\end{aligned}
$$

The partial safety factors in these equations can be expressed in terms of the statistical characteristics of the design variables:

$$
\begin{aligned}
& \gamma_{H_{s}}=\frac{H_{s}^{*}\left(P_{f}\right)}{H_{s}^{T}}=\frac{\mu_{H_{s}}^{N}-\alpha_{H_{s}} \sigma_{H_{s}}^{N} \beta_{T}}{H_{s}^{T}}=\frac{\mu_{H_{s}}^{N}}{H_{s}^{T}}\left(1-\alpha_{H_{s}} \delta_{H_{s}}^{N} \beta_{T}\right) \\
& \gamma_{R_{i}}=\frac{\mu_{R_{i}}}{R_{i}^{*}\left(P_{f}\right)}=\frac{\mu_{R_{i}}}{\mu_{R_{i}}+\alpha_{R_{i}} \sigma_{R_{i}} \beta_{T}}=\frac{1}{1+\alpha_{R_{i}} \delta_{R_{i}} \beta_{T}}
\end{aligned}
$$

where $\mu_{H_{s}}^{N}, \sigma_{H_{s}}^{N}$, and $\delta_{H_{s}}^{N}$ are the mean, standard deviation, and coefficient of variation, respectively, of the equivalent normal distribution of significant wave height.

On the other hand, Burcharth (1992) expressed the partial safety factors of load and resistance as follows:

$$
\begin{aligned}
& \gamma_{H_{s}}=\frac{H_{s}^{T_{P_{f}}}}{H_{s}^{T}}+\sigma_{F_{H s}}^{\prime}\left(1+\left(\frac{H_{s}^{3 T}}{H_{s}^{T}}-1\right) k_{\beta} P_{f}\right)+\frac{k_{s}}{\sqrt{P_{f} N}} \\
& \gamma_{R}=1-k_{\alpha} \ln P_{f}
\end{aligned}
$$

where $\sigma_{F_{H s}}^{\prime}$ is a parameter to account for the uncertainty of wave measurement (the 
larger the value, the larger the uncertainty), $H_{s}^{3 T}$ is the $3 T$-year return period value of $H_{s}$, and $H_{s}^{T_{P_{f}}}$ is the significant wave height corresponding to an equivalent return period $T_{P_{f}}$ defined as the return period corresponding to a probability $P_{f}$ that $H_{s}^{T_{P_{f}}}$ will be exceeded during the structural lifetime $T \cdot T_{P_{f}}$ is calculated from the encounter probability formula $T_{P_{f}}=\left(1-\left(1-P_{f}\right)^{1 / T}\right)^{-1} . N$ is the number of $H_{s}$ data used for fitting the extreme distributions, and $k_{\alpha}, k_{\beta}$, and $k_{s}$ are coefficients determined by an optimization procedure. For plunging waves, $k_{\alpha}=0.027, k_{\beta}=38$, and $k_{s}=0.05$. $N=50$ was used in Burcharth (1992).

The partial safety factor calculated by Eq. (30) does not consider the uncertainties involved in wave measurement and estimation of wave height distribution, which are represented by the second and third terms, respectively, on the right-hand side of Eq. (32) presented by Burcharth (1992). In order to compare the present result with the Burcharth's result, these two terms are added to Eq. (30) to give

$$
\gamma_{H_{s}}=\frac{H_{s}^{*}\left(P_{f}\right)}{H_{s}^{T}}+\sigma_{F_{H s}}^{\prime}\left(1+\left(\frac{H_{s}^{3 T}}{H_{s}^{T}}-1\right)^{k_{\beta} P_{f}}\right)+\frac{k_{s}}{\sqrt{P_{f} N}}
$$

Now, the Burcharth's method (Eq. (32)) and present method (Eq. (34)) for calculating $\gamma_{H_{s}}$ are different only in the definition of the wave height that causes failure. Burcharth assumes that failure occurs at the wave height of $H_{s}^{T_{P_{f}}}$, while the present study assumes that failure occurs at the wave height of $H_{s}^{*}\left(P_{f}\right)$, i.e., the wave height at the most probable failure point. In fact, $H_{s}^{*}\left(P_{f}\right)$ is smaller than $H_{s}^{T_{P_{f}}}$ by a few percent.

Table 4 shows the partial safety factors calculated by Burcharth (1992) on the 
assumption that all the design variables are uncorrelated. On the other hand, Table 5 shows the partial safety factors calculated by the present method for the cases of non-correlation and correlation. The resistance factors of non-correlation are almost same as the Burcharth's values, as they should be. The load factors, however, are slightly smaller than the Burcharth's values, because of the different definitions of wave height of failure.

Fig. 4 shows the partial safety factors of load and resistance calculated by the present method with and without considering the correlation. $\sigma_{F_{H S}^{\prime}}^{\prime}=0.05$ was used. The partial safety factors considering the correlation are slightly smaller than those of non-correlation for both load and resistance. The difference between correlation and non-correlation increases as the probability of failure decreases, especially for the resistance factor. This result corresponds with that in Fig. 3, in which the armor weight calculated by considering the correlation is smaller than that of non-correlation and the difference increases as the probability of failure decreases.

Table 4. Partial safety factors calculated by Burcharth (1992).

\begin{tabular}{ccccc}
\hline \multirow{2}{*}{$T($ year $)$} & $\left(P_{f}\right)_{T}$ & $\gamma_{R}$ & \multicolumn{3}{c}{$\gamma_{H_{s}}$} \\
\cline { 4 - 5 } & & & $\sigma_{F_{H s}^{\prime}}^{\prime}=0.05$ & $\sigma_{F_{H s}}^{\prime}=0.2$ \\
\hline \multirow{2}{*}{50} & 0.2 & 1.04 & 1.19 & 1.23 \\
& 0.1 & 1.06 & 1.29 & 1.37 \\
\multirow{2}{*}{100} & 0.2 & 1.04 & 1.18 & 1.22 \\
& 0.1 & 1.06 & 1.27 & 1.35 \\
\hline
\end{tabular}

Table 5. Partial safety factors calculated by present method.

\begin{tabular}{|c|c|c|c|c|c|c|c|}
\hline \multirow{3}{*}{$\begin{array}{c}T \\
\text { (year) }\end{array}$} & \multirow{3}{*}{$\left(P_{f}\right)_{T}$} & \multicolumn{2}{|c|}{$\gamma_{R}$} & \multicolumn{4}{|c|}{$\gamma_{H_{s}}$} \\
\hline & & \multirow{2}{*}{$\rho=0.0$} & \multirow{2}{*}{$\rho=0.51$} & \multicolumn{2}{|c|}{$\rho=0.0$} & \multicolumn{2}{|c|}{$\rho=0.51$} \\
\hline & & & & $\sigma_{F_{H s}}^{\prime}=0.05$ & $\sigma_{F_{H s}^{\prime}}^{\prime}=0.2$ & $\sigma_{F_{H s}^{\prime}}^{\prime}=0.05$ & $\sigma_{F_{H s}}^{\prime}=0.2$ \\
\hline \multirow{2}{*}{50} & 0.2 & 1.05 & 1.03 & 1.16 & 1.20 & 1.15 & 1.19 \\
\hline & 0.1 & 1.07 & 1.04 & 1.24 & 1.32 & 1.23 & 1.31 \\
\hline \multirow{2}{*}{100} & 0.2 & 1.05 & 1.03 & 1.14 & 1.19 & 1.13 & 1.18 \\
\hline & 0.1 & 1.07 & 1.04 & 1.21 & 1.30 & 1.20 & 1.28 \\
\hline
\end{tabular}



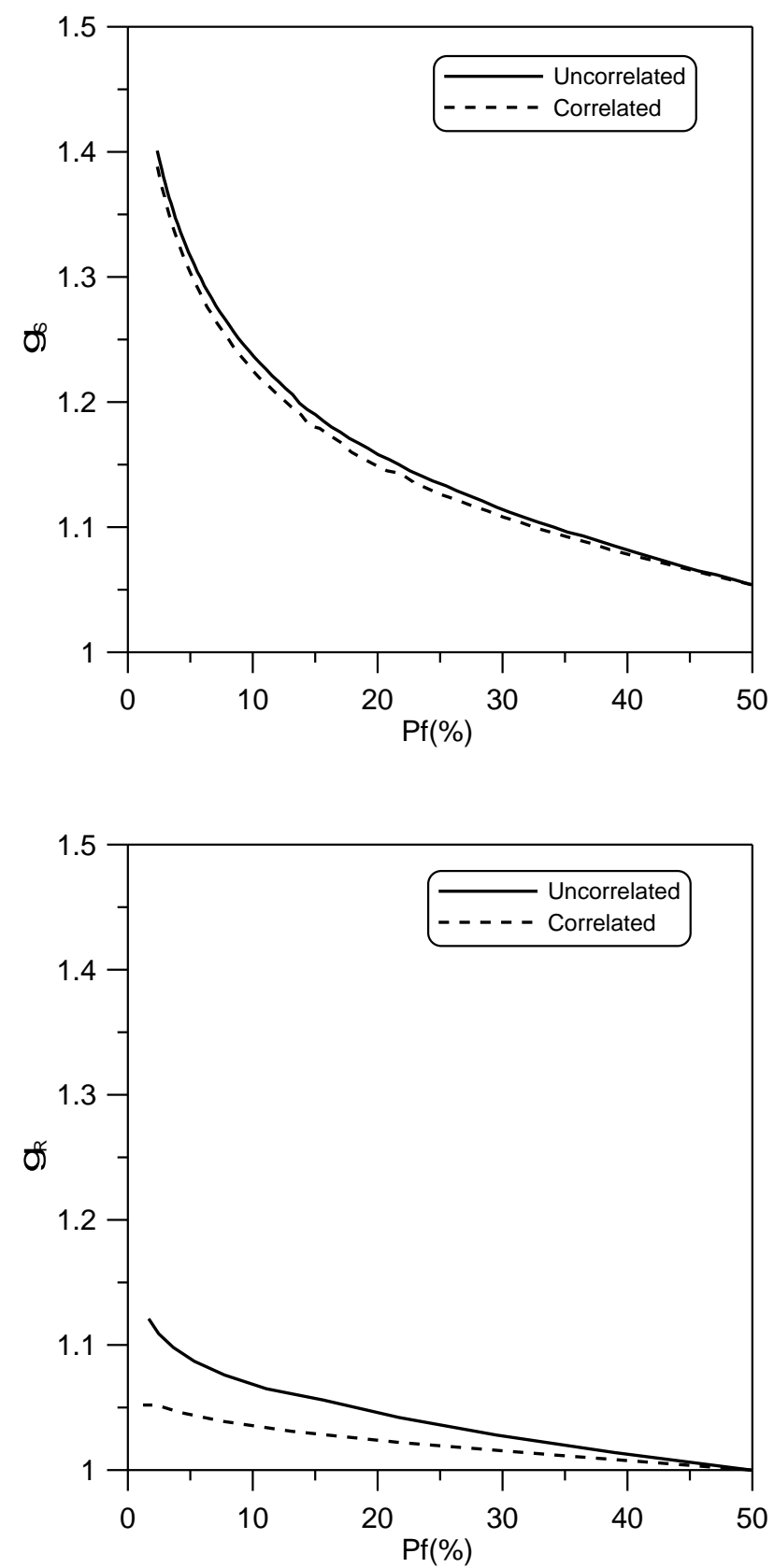

Fig. 4. Partial safety factors of load and resistance calculated by present method with $\rho=0.51 \quad(T=50$ years $)$. 


\section{Conclusion}

In this study, the partial safety factors of breakwater armor stones were calculated by considering the correlation between wave height and wave steepness. The correlation coefficient between these variables was calculated using the wave hindcasting data along the coasts of Korean peninsula. The probability of failure decreases if the correlation is taken into account. Therefore, a smaller weight of armor stones can be used if the correlation is taken into account in the design. The difference of the weight of armor stones between correlation and non-correlation increases as the probability of failure decreases. Therefore, more reduction of armor weight can be achieved as the design weight increases (or the probability of failure decreases). The partial safety factors calculated by considering the correlation are slightly smaller than those of non-correlation for both load and resistance. The difference increases as the probability of failure decreases, especially for the resistance factor. This result corresponds with that for the weight of armor stones. In summary, if the correlation is taken into account, the smaller partial safety factors and smaller armor stone weight are calculated, and the reduction becomes prominent as the probability of failure decreases (or the design weight increases). Lastly it should be mentioned that the correlation is not so important in the design of ordinary breakwaters for which the target probability of failure is several ten percent (See Fig. 4).

\section{Acknowledgments}

This work was supported by the Project for Development of Reliability-Based Design Methods for Port and Harbor Structures sponsored by Korea Ministry of Marine Affairs and Fisheries. This work was conducted at the Engineering Research Institute of Seoul National University. The authors thank Dr. Dong-Young Lee of Korea Ocean Research and Development Institute for providing the wave hindcasting data around Korean peninsula.

\section{References}

Burcharth, H. F. [1991] "Introduction of partial coefficient in the design of rubble mound 
breakwaters," Proc. Conf. Coastal Structures and Breakwaters, Institution of Civil Engineers, London, 543-565.

Burcharth, H. F. [1992] "Reliability evaluation of a structure at sea," Proc. Short Course on Design and Reliability of Coastal Structures, Venice, Scoula di S. Giovanni Evangelista.

Burcharth, H. F. \& Sørensen, J. D. [2000] "The PIANC safety factor system for breakwaters," Proc. Int. Conf. Coastal Structures, A. A. Balkema, Spain, 1125-1144.

Lee, D.-Y. \& Jun, K.-C. [2006] "Estimation of design wave height for the waters around the Korean peninsula," Ocean Science Journal 41, 245-254.

Rackwitz, R. [1976] "Practical probabilistic approach to design," Bulletin 112, Comite European du Beton, Paris, France.

van der Meer, J. W. [1987] "Stability of breakwater armor layers design formulae," Coastal Engineering 11, 219-239.

van der Meer, J. W. [1988] "Deterministic and probabilistic design of breakwater armor layers," J. Waterway, Port, Coastal, and Ocean Engineering 114, 66-80. 